\title{
A fractal analysis application of the pre-whitening technique to $\delta$ Scuti stars time series
}

\author{
S. de Franciscis, ${ }^{\star \star}$ J. Pascual-Granado ${ }^{\bullet},{ }^{\star}$ J. C. Suárez ${ }^{\odot},{ }^{1,2 \star}$ A. García Hernández ${ }^{\oplus},{ }^{2}$ \\ R. Garrido, ${ }^{1}$ M. Lares-Martiz ${ }^{1}$ and J. R. Rodón ${ }^{1}$ \\ ${ }^{1}$ Instituto de Astrofísica de Andalucía (IAA-CSIC), Glorieta de Astronomía s/n, E-18008 Granada, Spain \\ ${ }^{2}$ Departamento de Física Teórica y del Cosmos, Universidad de Granada (UGR), Av. de Fuente Nueva s/n, E-18071 Granada, Spain
}

Accepted 2019 June 3. Received 2019 May 31; in original form 2018 December 28

\begin{abstract}
Fractal fingerprints have been found recently in the light curves of several $\delta$ Scuti stars observed by Convection Rotation and planetary Transits (CoRoT) satellite. This sole fact might pose a problem for the detection of pulsation frequencies using classical pre-whitening techniques, but it is also a potentially rich source for information about physical mechanisms associated with stellar variability.

Assuming that a light curve is composed of a superposition of oscillation modes with a fractal background noise, in this work we applied the Coarse Graining Spectral Analysis (CGSA), a fast Fourier transform (FFT)-based algorithm, which can discriminate in a time series the stochastic fractal power spectra from the harmonic one.

We have found that the fractal background component is determining the frequency content extracted using classical pre-whitening techniques in the light curves of $\delta$ Scuti stars. This might be crucial to understand the amount of frequencies excited in these kinds of pulsating stars. Additionally, CGSA resulted to be relevant in order to extract the oscillation modes, this points to a new criterion to stop the pre-whitening cascade based on the percentage of fractal component in the residuals.
\end{abstract}

Key words: stars: activity-stars: oscillations - stars: variables: $\delta$ Scuti.

\section{INTRODUCTION}

$\delta$ Scuti stars exhibit rather complex oscillation spectra not yet fully understood.

These dense power spectra might be caused by the presence of a thin outer convective layer together with a rapid rotation. A correct mode identification could then be used to test theories of angular momentum transport and chemicals (Goupil et al. 2005).

It has also been suggested by Kallinger \& Matthews (2010) and Balona (2011) that the granulation background signal could be the origin of these light variation. Barceló Forteza et al. (2015) explored that resonant mode coupling can be an efficient mechanism to enhance or suppress a given mode. Neiner \& Lampens (2015) consider the existence of a magnetic field, and finally Lignières \& Georgeot (2009) predict a large number of chaotic modes in a non-spherical stellar model due to high rotation (recently confirmed by Barceló Forteza et al. 2017).
All these possible origins of the dense power spectra in $\delta$ Scuti stars are approximations to the problem from the physical point of view, nevertheless there are other issues to take into account from the data analysis perspective.

Ultraprecise data from Convection Rotation and planetary Transits (CoRoT; Baglin et al. 2006) and Kepler (Gilliland et al. 2010) have revealed that after a cleaning frequency process (commonly known in the asteroseismic community by pre-whitening) the residuals cannot be described by a white noise. This implies that, at some extent, the residuals are correlated with the original signal (see e.g. fig. 1 in Poretti et al. 2009). Similar pre-whitening methods based on the frequency domain, e.g. CLEAN algorithm (Roberts, Lehar \& Dreher 1987), have been popularized in radio astronomical images reconstruction, for which a simple minimization of the residuals is sought. This is not the case for the asteroseismic time series analysis because their set of frequencies has a clear physical meaning: the pulsation content of the physical system, which is the basis of asteroseismology. In a recent work (Pascual-Granado et al. 2018) a CoRoT $\delta$ Scuti light curves data set have been analysed using SIGSPEC, a pre-whitening algorithm, employed to obtain a rigorous statistical treatment of how to determine the significance of a peak in a discrete Fourier transform (DFT), finding, for each light curve,

\footnotetext{
^E-mail: sebast26@gmail.com (SdF); javier@iaa.es (JPG);

jcsuarez@ugr.es (JCS)
} 
thousands of significant frequencies, in particular for those stars with the grass of lower amplitudes phenomena. The origins of such grass effect might be detected in some way by fractal analysis, which may help to understand stochastic fluctuations and chaotic dynamic in the background spectra underlying the proper stellar pulsations modes. Recently we introduced a set of tools in order to investigate the fractal content of the light curves of $\delta$ Scuti stars (de Franciscis et al. 2018). These tools enable us to identify the non-harmonic content in the associated time series.

In this work, we assume that the light curve of $\delta$ Scuti stars can be considered as the sum of a background signal $y(t)$ and a harmonic function, representing the pulsating modes, i.e.

$f(t)=y(t)+\sum_{i=1}^{n} A_{i} \sin \left(2 \pi \omega_{i} t+\phi_{i}\right)$,

here $A_{i}, \omega_{i}$, and $\phi_{i}$ are, respectively, amplitude, frequency, and phase of the $i$ th mode.

We consider the background signal time series $y(t)$ as a self-affine curve (i.e. the generalization of fractal from geometrical objects to time series), which has the following property of non-homogeneous scaling relation (Malamud \& Turcotte 1999):

$y(t)=\lambda^{\mathrm{H}} y(\lambda t)$,

where $\mathrm{H}$ is the so-called Hausdorff exponent, characterizing longterm correlations and the type of self-affinity in time series. Equation (2) has to be taken in statistical meaning, so that the scaling relationship holds when one performs appropriate measures on mean values over pairs of points at the same distance or over equal length subseries or windows etc.

In this paper, we investigate how the non-harmonic component of the time series of a $\delta$ Scuti star can change the original concept of the pre-whitening technique. The relevance of this result is that we can discriminate the harmonic frequencies from spurious ones that can be assigned to a fractal component and may have a significant impact on asteroseismic studies.

The paper is organized as follows. Section 2 describes the observational data selected and how the classical pre-whitening technique is applied to them. Section 3 presents the results from applying Coarse Graining Spectral Analysis (CGSA), one of the fractal tools introduced in de Franciscis et al. (2018) to the selected data and a discussion in the asteroseismic context. Conclusions are summarized in Section 4.

\section{CoRoT DATA SET AND FREQUENCY EXTRACTION}

The sample used in this paper is the same as that in Pascual-Granado et al. (2018). From these 15 selected targets, two have been identified as hybrid $\gamma$ Dor- $\delta$ Scuti stars, i.e. HD 49434 (Chapellier et al. 2011) and HD 48784 (Barceló Forteza et al. 2017), while HD 172189 is an eclipsing binary (EB) system (Martín-Ruiz et al. 2005), and GSC 0144-03031 is a high-amplitude $\delta$ Scuti stars (HADS; Poretti et al. 2005), and the remaining are low-amplitude $\delta$ Scuti stars. In Table 1 the reader can find the physical parameters of every star in the sample. Other details can be found in Pascual-Granado et al. (2018).

As the power spectra are altered by the presence of gaps in the time series it was necessary to fill them up. The gap filling must be performed with an algorithm that preserves the frequency content of the original time series. This is guaranteed with the use of MIARMA algorithm as was demonstrated in the paper PascualGranado, Garrido \& Suárez (2015). In the aforementioned paper MIARMA algorithm works well including when the gaps are larger than the stellar pulsation periods. The algorithm is only limited by the amount of data contained in the segments bracketing the gaps. In the case of CoRoT data, there is about 10 per cent of data loss due to the satellite passing through the South Atlantic Anomaly, but this 10 per cent is spread along the observation interval and there are not many gaps bigger than a few hours, therefore, the conditions for an efficient interpolation using MIARMA are met.

Once obtained evenly spaced time series for every target, they were analysed using the popular numerical recipe SIGSPEC (Reegen 2007). This algorithm estimates the frequency content of the light curves by extracting a sequence of statistically significant frequencies. Detection is performed through a significance spectrum based on false alarm probability for each peak in the power spectrum. The iterative sequence stops when a significance threshold is reached [by default $\operatorname{sig}=5.0$, i.e. signal-to-noise ratio $(\mathrm{S} / \mathrm{N})=\approx 4]$. Here, we follow the procedure as in Pascual-Granado et al. (2018) using the same set of free parameters, in which a frequency interval of $2-100 \mathrm{~d}^{-1}$ was chosen.

Special considerations arose around two targets: the eclipsing binary HD 172189 and the HADS GSC 00144-03031. The transits of the eclipsing binary star HD 172189 introduce unknown effects that could bias the frequency analysis performed by classical pre-whitening techniques (Kopal 1977). Additionally, during the preparatory phase of this work we obtained results suggesting that the standard frequency analysis procedure for the HADS GSC 00144-03031 needs to be revised to include proper frequency combinations and harmonics in the pre-whitening (Lares-Martiz et al., in preparation). However, we decided to include both HD 172189 and GSC 00144-03031 in our analysis to verify if their results differ significantly from the other stars.

\section{RESULTS}

The CGSA splits in a time series the self-affine component and the harmonic one, giving as output the percentage of (stochastic) fractal power in time series (Yamamoto \& Hughson 1993). Here we perform a CGSA analysis (see Appendix A) on the residual time series output of our light curves sample. As can be seen in Figs $1-3$, where we study the first 150 pre-whitening steps in detail, in the vast majority of cases the CGSA of the residuals increase monotonically in the first tens steps, then reaching an asymptotic value. Nevertheless, extending the range of steps up to the whole pre-whitening output, up to hundreds and thousands of frequencies, the picture is very different, in the most of the cases the curves still grow, or grow and decrease with a long tail. Figs 4-6 show the CGSA of the residual time series at every 50 steps of the prewhitening in the order given by SIGSPEC. It is possible to observe that CGSA percent Frac of the residual time series has different evolutions with the number of step $n$ depending from the star under examination. The increasing monotonic behaviour and the reaching of a plateau in percent $\operatorname{Frac}(n)$ (Figs 4 and 6) are a good indication of the mechanism by which SIGSPEC extracts step by step the modes, increasing the 'fractality' of the resulting residual series, until the plateau, where all the oscillating modes have been already extracted. For those anomalous stars where per cent $\operatorname{Frac}(n)$ has a decreasing cue, showing a steep initial increase with a maximum of CGSA in the first 150-200 first steps followed by a decreasing tail, SIGSPEC appears to be extracting modes in residuals containing only 
Table 1. Coarse Grained Spectral Analysis (CGSA) per cent Frac for the sample of 15 CoRoT $\delta$ Scuti stars studied. Calculated on the original time series gap filled with MIARMA algorithm.

\begin{tabular}{|c|c|c|c|c|c|c|c|c|}
\hline ID & $\begin{array}{l}\text { CGSA } \\
q \text {-Frac }\end{array}$ & $\mathrm{SpT}$ & $\begin{array}{l}T_{\text {eff }} \\
(\mathrm{K})\end{array}$ & $\log g$ & $\begin{array}{l}v \sin i \\
(\mathrm{mag})\end{array}$ & $\begin{array}{c}\text { PulsT } \\
\left(\mathrm{km} \mathrm{s}^{-1}\right)\end{array}$ & Obs. time & $\begin{array}{c}\text { Run } \\
\text { (d) }\end{array}$ \\
\hline GSC 00144-03031 & 0.031 & A8 & $7822 \pm 400$ & $3.41 \pm 0.32$ & & HADS & 79.133 & IRLRa04 \\
\hline HD 172189 & 0.336 & $\mathrm{~A} 6 \mathrm{~V}$ & $7600 \pm 150$ & $3.48 \pm 0.08$ & $78 \pm 3$ & $\delta$ Sct $(\mathrm{EB})$ & 149.013 & LRc02 \\
\hline HD 174532 & 0.070 & A2 & $6783 \pm 228$ & 3.684 & 32 & $\delta$ Sct & 26.239 & $\mathrm{SRc} 02$ \\
\hline HD 174589 & 0.032 & F3III & $7359 \pm 251$ & $3.88 \pm 0.14$ & 101 & $\delta \mathrm{Sct}$ & 26.168 & $\mathrm{SRc} 02$ \\
\hline HD 174936 & 0.117 & $\mathrm{~A} 2$ & $8000 \pm 200$ & $4.08 \pm 0.2$ & 169.7 & $\delta$ Sct & 27.194 & $\mathrm{SRc} 01$ \\
\hline HD 181555 & 0.062 & A5V & $7000 \pm 200$ & $4.3 \pm 0.2$ & 200 & $\delta$ Sct & 156.645 & LRc01 \\
\hline HD 41641 & 0.067 & A5V & 7561 & 3.71 & 28 & $\delta$ Sct & 94.432 & IRLRa05 \\
\hline HD 48784 & 0.224 & F0 & $6990 \pm 140$ & & 108 & Hybrid & 25.305 & SRa05 \\
\hline HD 49434 & 0.235 & F1IV & $7632 \pm 126$ & $4.43 \pm 0.2$ & $85.7 \pm 4.3$ & Hybrid & 136.890 & LRa01 \\
\hline HD 50844 & 0.055 & A2 & $7400 \pm 200$ & $3.6 \pm 0.2$ & $58 \pm 2$ & $\delta \mathrm{Sct}$ & 57.713 & $\mathrm{IRa01}$ \\
\hline HD 50870 & 0.019 & A8III & $7660 \pm 250$ & $3.68 \pm 0.25$ & $37.5 \pm 2.5$ & $\delta$ Sct & 114.413 & LRa02 \\
\hline
\end{tabular}

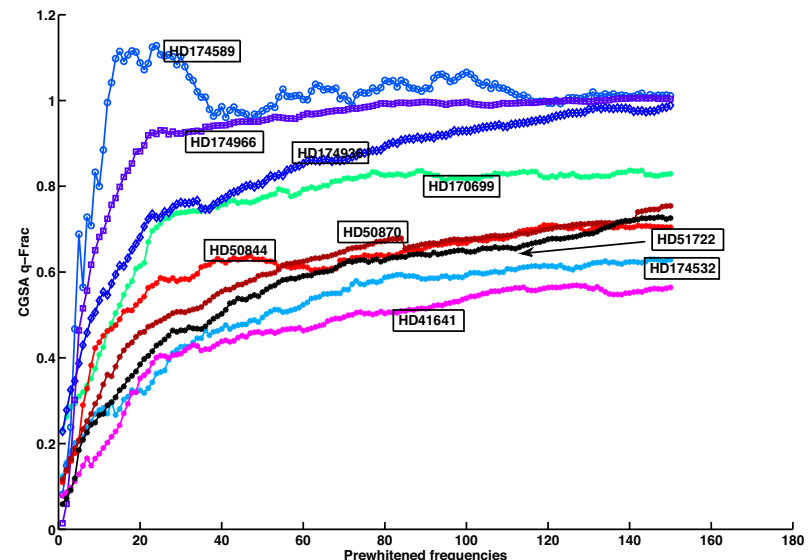

Figure 1. $q$-Frac CGSA versus pre-whitening step for $\delta$ Scuti stars data base under examination. First 150 steps are represented here.

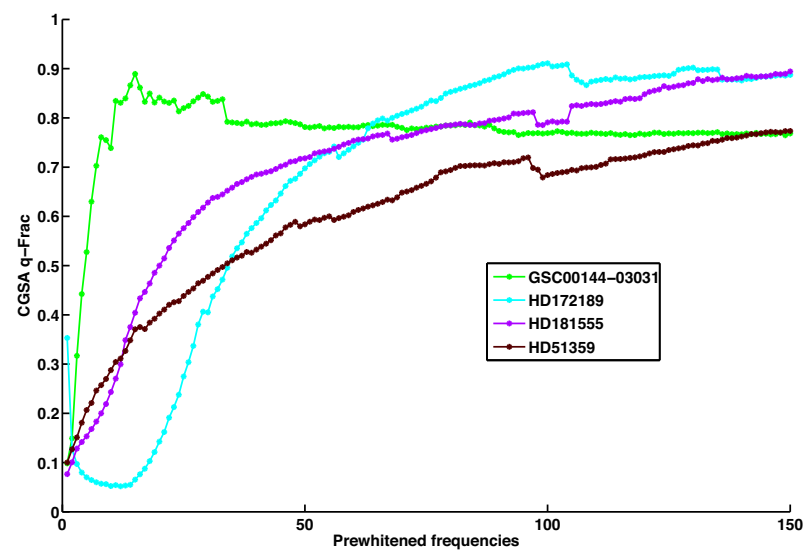

Figure 2. $q$-Frac CGSA versus pre-whitening step for $\delta$ Scuti stars data base samples with anomalous pre-whitening cascade. First 150 steps are represented here. Colour scheme is the same as in Fig. 1.

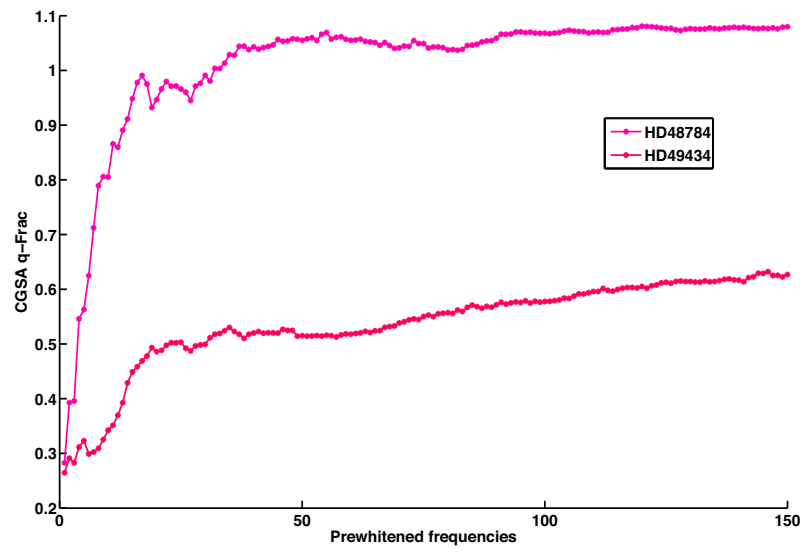

Figure 3. $q$-Frac CGSA versus pre-whitening step for Gamma Doradus stars data base samples. First 150 steps are represented here. Colour scheme is the same as in Fig. 1.

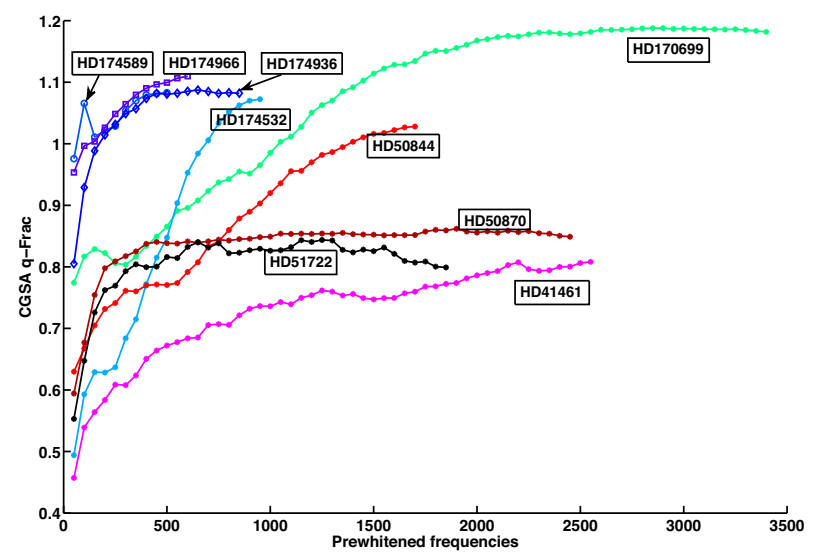

Figure 4. $q$-Frac CGSA versus pre-whitening step for the $\delta$ Scuti stars samples. Points are separated by 50 pre-whitening steps. 


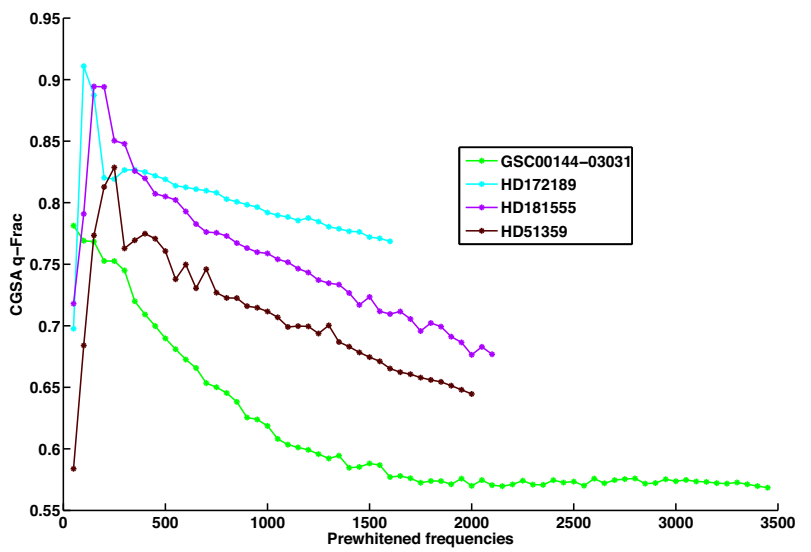

Figure 5. $q$-Frac CGSA versus pre-whitening step for the $\delta$ Scuti stars samples with anomalous pre-whitening cascade. Points are separated by 50 pre-whitening steps.

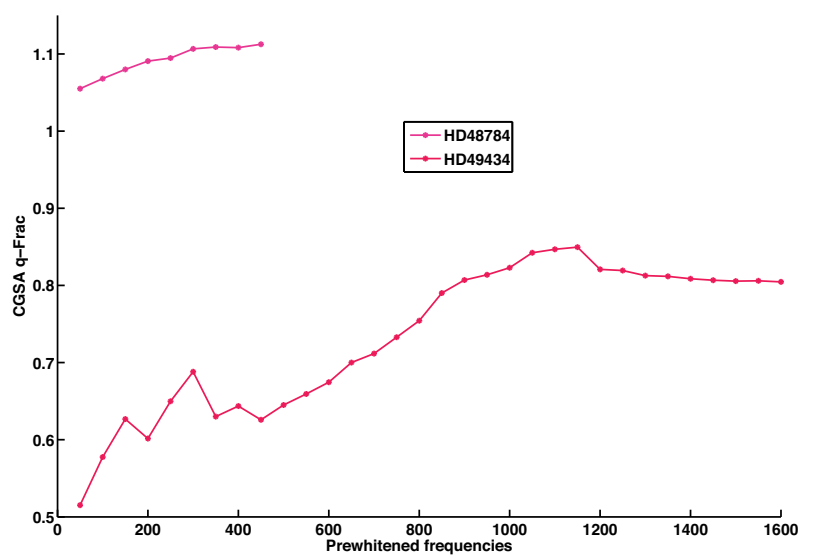

Figure 6. $q$-Frac CGSA versus pre-whitening step for the Gamma Doradus stars samples. Points are separated by 50 pre-whitening steps.

background noise, i.e. introducing a new artificial signal (Fig. 5). This explains why the pre-whitening process causes a cascade of spurious frequencies (Balona 2014). This shows that this new prewhitening criterion is more robust for extracting the sinusoidal components of the light curves.

A first attempt to introduce a novel stop criterion for SIGSPEC could be to study percent $\operatorname{Frac}(n)$ and establish where it reaches the plateau. Nevertheless, maximum value of per cent Frac depends on the self-affine exponent of noise signal (see appendix A3 in de Franciscis et al. 2018), and moreover local fluctuations are perturbing the study of the first derivative of those curves. In Table 2 we collect the values for the critical step in the pre-whitening cascade determined according to a CGSA-based criterion. In order to reduce the fluctuation we applied to the CGSA curves a five-point moving average filter. Then, several approaches were tried to find the best estimation of the critical step (i.e. stop criterion). It was found that most of the CGSA curves can be fitted optimally with a hyperbolic tangent function. Since this function has no critical points and their derivatives are smooth functions, we finally used an epsilon around the limit value of CGSA to determine whether the critical step is reached. We have found empirically in our tests that $\operatorname{CGSA}(N)-\operatorname{CGSA}(i)<\epsilon=0.02$ (where $\operatorname{CGSA}(N)$ is the last value) is a robust value.

This procedure is valid only for those cases where CGSA is a strictly monotonically increasing function. If this function has a maximum and then decreases this points to an anomaly that is caused by the lack of convergence of the pre-whitening cascade to an uncorrelated Gaussian noise. We previously mentioned that an anomalous frequency analysis might be related to unknown effects introduced by a binary component of the stellar system (e.g. HD 172189), by the non-linear response of the exciting mechanism (e.g. GSC 00144-03031), or by the spectrum modulation from the significant deformation of a fast rotating star (e.g. HD 181555). This check proves the robustness of the method.

However, note that in Fig. 2 HD 172189 is different to the other anomalous cases showing a decrease in the $q$-Frac curve during the first $\sim 20$ steps. This might be related to the harmonic fitting of the eclipses, which is not an intrinsically sinusoidal signal. As a consequence the non-linear least-squares fitting performed by SIGSPEC introduces a harmonic contribution to the residuals that produce a decrease in the $q$-Frac. This suggests a possible procedure to detect transits by studying the CGSA $q$-Frac during the first steps of the pre-whitening.

Finally, regarding the last anomalous case HD 51359, our study could be a hint showing that this star belongs to one of the aforementioned cases, but there is no clue in the literature about this, so complementary observations are necessary to confirm this point.

It is also worth mentioning that HD 41641 has rotational modulation produced by spots as shown in Escorza et al. (2016) but, since the $v \sin i$ (see Table 1) is considerably slow, contrary to HD 181555 we do not expect HD 41641 to be an anomalous case.

The number of frequencies extracted at the critical step shown in Table 2 can be compared with the total number of frequencies extracted at the end of the pre-whitening cascade (Nf), i.e. when SIGSPEC stops, and the number of frequencies found in the literature. Notice that in most of the cases the number of frequencies extracted at the critical step is more similar to the value shown in the literature. However, the comparison with the literature must be done carefully since different criteria are used in different papers, e.g. in García Hernández et al. (2013) 185 frequencies were extracted up to $\operatorname{sig}=10.0$, but we have used the default value sig $=5.0$, which is more similar to the typical $\mathrm{S} / \mathrm{N}$ equal to 4 . Therefore, the comparison with $\mathrm{Nf}$ is more reliable since we used a homogeneous procedure for the whole sample of stars. Our stop criterion gives a reduced set of frequencies containing from 16 per cent (HD 50870) to 89 per cent (HD 174532) of the frequencies extracted with a classical pre-whitening.

Notice also the values shown in columns 6 and 7 for the significance and $\mathrm{S} / \mathrm{N}$ corresponding to the last step before stop. These lead to a star-dependent criterion going from the more usual value of 4.8 for HD 174532 to a much more conservative value of 20.1 for HD 51722. In Baran, Koen \& Pokrzywka (2015) it is shown that the classical $\mathrm{S} / \mathrm{N}>4$ criterion from Breger et al. (1993) is insufficient since the confidence level depends on the number of data points. Here, in contrast, we show that the significance limit is very dependent on the signal itself. Our CGSA criterion is also consistent with Sodor et al. (in preparation) where they found that for $\mathrm{S} / \mathrm{N}<30$ unmatched frequencies can appear in the results obtained using different algorithms. In addition, CGSA provides a useful criterion to remove spurious frequencies from the extracted set without the necessity of performing any 
Table 2. Critical values for the pre-whitening process for each star analysed. If the CGSA is increasing monotonically, a critical step is reached when the CGSA fraction approximates an asymptotic value within a certain tolerance interval. Second column shows the number of frequencies detected when the pre-whitening process reach the critical step. Third column shows the corresponding CGSA limit value. Fourth column shows the total number of frequencies extracted at the end of the pre-whitening cascade (i.e. when SigSpec stops). Fifth column shows the number of frequencies extracted in the references cited below. Finally, the sixth and last columns show the significance and its corresponding signal-to-noise ratio (S/N) values reached at the critical step. ${ }^{1}$ García Hernández et al. (2009); ${ }^{2}$ García Hernández et al. (2013); ${ }^{3}$ Escorza et al. (2016); ${ }^{4}$ Barceló Forteza et al. (2017); ${ }^{5}$ Chapellier et al. (2011); ${ }^{6}$ Poretti et al. (2009); ${ }^{7}$ Mantegazza et al. (2012).

\begin{tabular}{lcccccc}
\hline Star & Step & CGSA & Nf & Literature & Sig & S/N \\
\hline HD 170699 & 2000 & 1.1677 & 3442 & - & 15.499 & 6.742 \\
HD 174532 & 850 & 1.0625 & 951 & - & 7.854 & 4.799 \\
HD 174589 & 100 & 1.0656 & 503 & - & 107.471 & 17.753 \\
HD 174936 & 400 & 1.0735 & 870 & $422^{1}$ & 16.181 & 6.889 \\
HD 174966 & 400 & 1.0902 & 647 & $185^{2}$ & 8.693 & 5.049 \\
HD 41641 & 2050 & 0.7900 & 2550 & $90^{3}$ & 20.759 & 7.802 \\
HD 48784 & 250 & 1.0947 & 464 & $163^{4}$ & 11.769 & 5.875 \\
HD 49434 & 850 & 0.7901 & 1612 & $840^{5}$ & 77.578 & 15.083 \\
HD 50844 & 1450 & 1.0104 & 1746 & $1000^{6}$ & 9.818 & 5.366 \\
HD 50870 & 400 & 0.8374 & 2484 & $734^{7}$ & 119.303 & 18.704 \\
HD 51722 & 300 & 0.7929 & 1693 & - & 137.947 & 20.113 \\
\hline
\end{tabular}

simulation or analytical approximation to determine the significance level.

Last column of Table 1 lists the corresponding CoRoT run for each observation. For example, stars HD 174532 and HD 174589 have been observed during the same run ( $\mathrm{SRc02}$ ), i.e. they have the same spectral window. However, in Table 2 the CGSA results for these stars are much different. While for HD 174532 the CGSA stop criterion reduces the number of frequencies from 951 to 850 , for HD 174589 the number goes from 503 down to just 100 . Therefore, observational noise (e.g. instrumental effects) is not the most significant factor but the pre-whitening procedure itself that is causing the injection of spurious signal.

In order to better understand the CGSA behaviour with respect the pre-whitening step we build a controlled theoretical experiment, i.e. a toy model of a typical $\delta$ Scuti stars light curve. This model is based on our main assumption of equation (1), thus the light curve of HD 174523 is built by summing sinusoidal functions with amplitudes, frequencies, and phases obtained from the last pre-whitening step, i.e. the oscillation spectrum, together with a Gaussian white noise, with statistical properties (mean and standard deviation) similar to those given by light-curve residuals.

The toy model representative of HD 174523 has an analogue CGSA curves (Fig. 7), although not identical. This is somewhat expected since the only source of fractal behaviour included in the model is the Gaussian white noise, whereas the observed light curves may have more (e.g. turbulence, convection, etc.). Nevertheless, Anderson-Darling (A-D) test performed on the frequency distributions of original and toy model shows that the distributions of toy model and data are compatible along the whole pre-whitening iterations (Pascual-Granado et al. 2018). This evidence is consistent with previous results obtained for this star, since the critical step is more compatible with SIGSPEC than in other cases. An ongoing work is aimed to extend this study to the whole data set.

Finally, several hypotheses are compatible with a fractal background signal: granulation, magnetic cycles, rotational effects, etc. In any case, this method allows us to extract the harmonic components of the light curve reducing considerably the set of

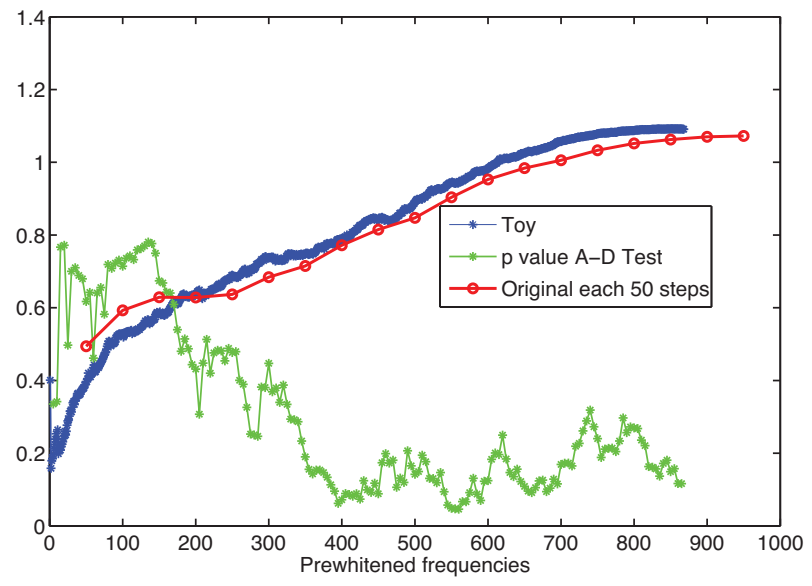

Figure 7. $q$-Frac CGSA versus pre-whitening step for HD 174532 and its toy model.

frequencies found when performing a classical pre-whitening, this is due to the lack of a precise criteria to stop the peak bagging. In a forthcoming paper, we will explore the impact of these technique comparing directly the frequencies found to be harmonic with those from theoretical models. The stop criterion suggested here for the pre-whitening procedure might be implemented easily in any frequency analysis package. In particular, CGSA will be implemented on a new version of SIGSPEC that avoids failing to stop when no harmonic component is left in the residuals.

\section{CONCLUSIONS AND FUTURE PROSPECTS}

We have performed a systematic fractal analysis to every single step of the pre-whitening procedure applied to the well-characterized sample of $\delta$ Scuti stars of CoRoT seismo field. Our basic hypothesis is that light curves are composed of a superposition of proper (harmonic) modes, together with a typical fractal background noise. We applied the CGSA algorithm to discriminate the stochastic fractal power spectra from the harmonics. We have found a self- 
consistent stop criterion for pre-whitening-type methods widely used in asteroseismic frequency analyses.

This new method is robust since it does not require any hypothesis about the non-harmonic component of the signal, and more interestingly, it does not rely on the $\mathrm{S} / \mathrm{N}$ anymore. Indeed we found a wide diversity of $\mathrm{S} / \mathrm{N}$ corresponding to the CGSA criterion counterparts, ranging from 4.8 to 20.1 for the sample of $\delta$ Scuti stars studied. This latter results go in the same direction of recent works (e.g. Baran et al. 2015) claiming that $\mathrm{S} / \mathrm{N}$ criterion cannot longer be trusted.

This work gives support to Pascual-Granado et al. (2015) hypothesis about the fractal origin of the non-analyticity found in the functions underlying the asteroseismic time series observed with CoRoT and Kepler. This work gives us arguments and tools to tackle this problem.

In order refine and better assess the whole potential of the method, we need to evaluate it on a large sample of stars observed with different instruments (work in progress).

In the next steps of our research line we would improve our fractal analysis tools in order to discriminate between fractal part originated from chaotic dynamic and fractal part coming from self-affine stochastic fluctuations. About the possible physical and non-physical (i.e. instrumental effects) origins of these two dynamics, we remind to a more deep study of instrument technical specifications.

\section{ACKNOWLEDGEMENTS}

The authors would like to thank the anonymous referee for their valuable comments that helped to improve considerably the manuscript. SdF, JP-G, JRR, ML-M, and RG acknowledge financial support from the State Agency for Research of the Spanish MCIU through the 'Center of Excellence Severo Ochoa' award for the Instituto de Astrofísica de Andalucía (SEV-2017-0709). SdF, JP-G, JRR, ML-M, and RG acknowledge funding support from Spanish public funds for research under projects ESP2015-65712-C5-3R. JCS and AGH acknowledge funding support from Spanish public funds for research under projects ESP2017-87676-2-2 and ESP2015-65712-C5-5-R. SdF, JP-G, and RG acknowledge support from the 'Junta de Andalucía' regional government under project 2012-P12-TIC-2469. JCS also acknowledges funding support from project RYC-2012-09913 under the 'Ramón y Cajal' program of the Spanish MINECO. This study is based on data from the CoRoT archive at Centro de Astrobiología (CAB). AGH, JCS, and JP-G acknowledge the International Space Science Institute (ISSI) for supporting the SoFAR international team. ${ }^{1}$

\section{REFERENCES}

Baglin A. et al., 2006, 36th COSPAR Scientific Assembly. Meeting abstract from the CDROM, \#3749

Balona L. A., 2011, MNRAS, 415, 1691

Balona L. A., 2014, MNRAS, 439, 3453

Baran A. S., Koen C., Pokrzywka B., 2015, MNRAS, 448, L16

Barceló Forteza S., Michel E., Roca Cortés T., García R. A., 2015, A\&A, 579, A133

Barceló Forteza S., Roca Cortés T., García Hernández A., García R. A., 2017, A\&A, 601, A57

Breger M. et al., 1993, A\&A, 271, 482

${ }^{1}$ http://www.issi.unibe.ch/teams/sofar/
Chapellier E. et al., 2011, A\&A, 525, A23

de Franciscis S., Pascual-Granado J., Suárez J. C., García Hernández A., Garrido R., 2018, MNRAS, 481, 4637

Escorza A. et al., 2016, A\&A, 588, A71

García Hernández A. et al., 2009, A\&A, 506, 79

García Hernández A. et al., 2013, A\&A, 559, A63

Gilliland R. L. et al., 2010, ApJ, 713, L160

Goupil M.-J., Dupret M. A., Samadi R., Boehm T., Alecian E., Suarez J. C.,

Lebreton Y., Catala C., 2005, J. Astrophys. Astron., 26, 249

Kallinger T., Matthews J. M., 2010, ApJ, 711, L35

Kopal Z., 1977, Ap\&SS, 46, 87

Lignières F., Georgeot B., 2009, A\&A, 500, 1173

Malamud B. D., Turcotte D. L., 1999, J. Stat. Planning Inference, 80, 173

Mantegazza L. et al., 2012, A\&A, 542, A24

Martín-Ruiz S., Amado P. J., Suárez J., Moya A., Arellano Ferro A., Ribas I., Poretti E. A., 2005, A\&A, 440, 711

Neiner C., Lampens P., 2015, MNRAS, 454, L86

Pascual-Granado J., Garrido R., Suárez J., 2015, A\&A, 575, A78

Pascual-Granado J., Suárez J., Garrido R., Moya A., García Hernández A., Rodón J., Lares-Martiz M., 2018, A\&A, 614, A40

Poretti E. et al., 2005, A\&A, 440, 1097

Poretti E. et al., 2009, A\&A, 506, 85

Reegen P., 2007, A\&A, 467, 1353

Roberts D. H., Lehar J., Dreher J. W., 1987, AJ, 93, 968

Yamamoto Y., Hughson R. L., 1993, Phys. D: Nonlinear Phenomena, 68 , 250

\section{APPENDIX A: CGSA METHOD}

CGSA is based on the consideration that in a self-similar time series the fast Fourier transform (FFT) phases follow a uniform distribution $\Theta_{k} \in[0,2 \pi]$. We consider the original time series $y(i)$ and the series obtained by scaling $y(i)$ by a factor of 2 and $1 / 2$ :

$y_{2}=\{y(2), y(4), y(6), \ldots\}$,

$y_{1 / 2}=\{y(1), y(1), y(2), y(2), \ldots\}$.

Next we cut those series in $N_{\mathrm{s}}$ partially overlapping subsets, each one having size the 90 per cent of the total length. ${ }^{2}$ For each window $m$ we compute the autopower spectrum $S_{y y, m}$ and the cross-power spectrum $^{3}$ between the original series and the rescaled ones, i.e. $S_{y y_{2}, m}$ and $S_{y y_{\frac{1}{2}}, m}$. If $y(i)$ is constituted by a sum of a few harmonics with fixed phase relationship, it is possible to exploit the phase difference between windows $m-2$ and $m-1$ to orthogonalize $S_{y y_{z}, m}$, where $z \in\{1 / 2,2\}$, with the rotating factor

$S_{y y_{z}, m}^{\mathrm{o}}(k)=S_{y y_{z}, m}(k) \mathrm{e}^{-\mathrm{i}\left[\pi / 2-\left(\Theta_{m-1, k}-\Theta_{m-2, k}\right)\right]}$.

The residuals of such orthogonalization process are non-zero in selfaffine series, because any rescaled harmonic will find its counterpart in the original series and the phase relationships are always randomly distributed. Taking advantage of Schwartz's inequality we can calculate the fractal module cross-correlations:

$$
\begin{aligned}
& \left\langle\left\|S_{y y_{z}, m-1}^{\mathrm{frac}}(k)\right\|\right\rangle_{m} \equiv \frac{\left\langle\left\|S_{y y_{z}, m-1}(k) \cdot S_{y y_{z}, m}^{\mathrm{o}}(k)\right\|\right\rangle_{m}}{\left\langle S_{y y_{z}, m-1}(k)\right\rangle_{m}} \\
& \leq\left\langle\left\|S_{y y_{z}, m}^{\mathrm{o}}(k)\right\|\right\rangle_{m} .
\end{aligned}
$$

\footnotetext{
${ }^{2} N_{\mathrm{S}}$ is an additional free parameter.

${ }^{3}$ Cross-power spectrum is defined as the Fourier transform of the crosscorrelation function, i.e. $S_{X Y}(k)=\sum_{n} \sum_{n^{\prime}} X(n) Y\left(n+n^{\prime}\right) \mathrm{e}^{\mathrm{i} k n}$.
} 
Finally, considering the possible distortions that could emerge by the finite size of the original series and the coarse graining of $y_{2}$ and $y_{1 / 2}$, we define the fractal power and the percentage of fractal power as

$\left\|S^{\mathrm{frac}}(k)\right\| \equiv \sqrt{\left\|S_{y y_{2}}^{\mathrm{frac}}(k)\right\| \cdot\left\|S_{y_{\frac{1}{2}}}^{\mathrm{frac}}(k)\right\|}$,

$q-$ Frac $\equiv \frac{\sum_{k}\left\|S^{\mathrm{frac}}(k)\right\|}{\sum_{k}\|S(k)\|}$.
SIGSPEC algorithm works essentially by extracting at each step the mode with the highest significance from the residual time series. In this way the sequence of modes is ordered by their power spectra amplitude. For each step of the pre-whitening, the CGSA is computed for the corresponding residuals, measuring the fractality percentage of each time series and measuring how the self-affine background weight evolves along the pre-whitening cascade.

This paper has been typeset from a $\mathrm{T}_{\mathrm{E}} \mathrm{X} / \mathrm{L} \mathrm{T} \mathrm{E} \mathrm{X}$ file prepared by the author. 